\title{
The Nopp140 gene in Drosophila melanogaster displays length polymorphisms in its large repetitive second exon
}

\author{
Sonu Shrestha Baral $^{1} \cdot$ Patrick J. DiMario ${ }^{1}$ (D) \\ Received: 19 January 2019 / Accepted: 10 April 2019 / Published online: 20 April 2019 \\ (c) The Author(s) 2019
}

\begin{abstract}
Nopp140, often called the nucleolar and Cajal body phosphoprotein (NOLC1), is an evolutionarily conserved chaperone for the transcription and processing of rRNA during ribosome subunit assembly. Metazoan Nopp140 contains an amino terminal LisH dimerization domain and a highly conserved carboxyl domain. A large central domain consists of alternating basic and acidic motifs of low sequence complexity. Orthologous versions of Nopp140 contain variable numbers of repeating basic-acidic units. While vertebrate Nopp 140 genes use multiple exons to encode the central domain, the Nopp140 gene in Drosophila uses exclusively exon 2 to encode the central domain. Here, we define three overlapping repeat sequence patterns ( $\mathrm{P}, \mathrm{P}^{\prime}$, and $\left.\mathrm{P}^{\prime \prime}\right)$ within the central domain of D. melanogaster Nopp140. These repeat patterns are poorly conserved in other Drosophila species. We also describe a length polymorphism in exon 2 that pertains specifically to the $\mathrm{P}^{\prime}$ pattern in D. melanogaster. The pattern displays either two or three 96 base pair repeats, respectively, referred to as Nopp140-Short and Nopp140-Long. Fly lines homozygous for one or the other allele, or heterozygous for both alleles, show no discernible phenotypes. PCR characterization of the long and short alleles shows a poorly defined, artifactual bias toward amplifying the long allele over the short allele. The significance of this polymorphism will be in discerning the largely unknown properties of Nopp140's large central domain in rDNA transcription and ribosome biogenesis.
\end{abstract}

Keywords Nopp140 $\cdot$ Repeat polymorphism $\cdot$ Drosophila melanogaster

\section{Introduction}

The nucleolar phosphoprotein of $140 \mathrm{kDa}(\mathrm{Nopp} 140)$ is a ribosome assembly factor that locates within the dense fibrillar component of nucleoli and within nuclear Cajal bodies (CBs) (Meier and Blobel 1990, 1992). It was first described as a chaperone that shuttles between the nucleolus and cytoplasm (Meier and Blobel 1992), perhaps to facilitate the import of other ribosome assembly factors that have SV40 T

Communicated by S. Hohmann.

Electronic supplementary material The online version of this article (https://doi.org/10.1007/s00438-019-01568-6) contains supplementary material, which is available to authorized users.

Patrick J. DiMario

pdimari@1su.edu

Sonu Shrestha Baral

sshre22@1su.edu

1 Department of Biological Sciences, Louisiana State University, Baton Rouge, LA 70803, USA antigen-type nuclear localization signals (Meier and Blobel 1990). Subsequent work showed that Nopp140 associates with C/D box small nucleolar ribonucleoprotein (snoRNPs) that guide nucleotide-specific 2'-O-methylation of the pre-rRNA, and with H/ACA box snoRNPs that direct site-specific pseudouridylation of pre-rRNA and snRNAs (Yang et al. 2000). These interactions with Nopp140 may initiate within CBs as Nopp140 is thought to shuttle these snoRNPs from CBs to nucleoli (Isaac et al. 1998; Yang et al. 2000; Wang et al. 2002; Meier 2005; Lo et al. 2006; He and DiMario 2011). Nopp140 also interacts with RNA Pol I to regulate rRNA transcription, perhaps linking RNA Pol I transcription with pre-rRNA processing (Chen et al. 1999; Meier 2005).

While metazoan Nopp140 orthologues vary in size, they are conserved in overall domain organization (Meier and Blobel 1992; Cairns and McStay 1995; Meier 1996; Kelly et al. 2006; Lee et al. 2014). The amino terminus consists of a LisH dimerization domain (Mateja et al. 2006), followed by a large central domain with variable numbers of alternating basic and acidic peptide motifs. With one basic motif and one adjacent acidic motif forming a unit, human 
Nopp140 has 11 repeat units, Xenopus laevis Nopp180 has 17 repeat units (Cairns and McStay 1995), and C. elegans Nopp140 (dao-5) has 27 repeat units (Lee et al. 2014). The basic motifs are rich in lysine, alanine, and proline while the acidic motifs are rich in aspartate, glutamate, and serine. These serine residues are phosphorylated in vivo by casein kinase type II (CKII) enzymes thus contributing to the acidic properties of the motif (Meier 1996; Li et al. 1997). The precise function of this central domain remains largely unknown. The carboxyl domain is the most conserved region among the Nopp140 orthologues. While its function also remains poorly understood, a conserved but putative protein kinase A phosphorylation site within the carboxyl domain indicates that the carboxyl domain is involved in regulatory signaling events (Meier 1996).

Human Nopp140, also called nucleolar and Cajal body phosphoprotein (NOLC1; CCDS 65925.1), is encoded by NOLCl (ID: 9221) which maps to chromosome 10 (NC_000010.11). As an example of vertebrate Nopp140 gene organization, NOLCl contains 13 exons that encode isoforms of 700 or 707 amino acids. Exon 1 encodes the LisH domain, and exon 2 encodes the remaining 18 residues of the amino terminal domain. Exons 3-11 encode the large central domain that contains the 11 basic-acidic repeat units; some of the individual basic or acidic motifs span exon junctions. Exons 12 and 13 in NOLCl encode the conserved carboxyl tail of 83 amino acids. No mutations are known to exist for human $N O L C l$, suggesting de novo mutations in NOLCl are embryonic lethal. On the other hand, dao-5 in C. elegans is encoded by 5 exons. Exon 3 and the relatively large exon 4 encode the majority of the 27 basic-acidic repeat units, while the $3^{\prime}$ end of exon 4 and exon 5 encode the well-conserved carboxyl domain. At least one mutation in dao-5 has been described (Lee et al. 2014).

Treacle is another nucleolar ribosome biogenesis factor related to Nopp140 in structure and function, but thus far found only in vertebrates. Human treacle is encoded by the TCOF1 gene (gene ID: 6949; chromosome 5, NC_000005.10); haplo-insufficiency mutations in TCOF1 result in the Treacher Collins syndrome (TCS), a ribosomopathy in which select neural crest cells undergo p53-dependent apoptosis resulting in impaired craniofacial development (Jones et al. 2008; Sakai and Trainor 2009; Narla and Ebert 2011). Similar to NOLC1, the TCOF1 gene consists of multiple exons. Exon 1 again encodes a LisH domain, and similar to mammalian Nopp140, treacle contains several basic-acidic repeats comprising a large central domain (Wise et al. 1997). As with human NOLC1, multiple exons in the TCOF1 gene encode these repeats (Isaac et al. 2000).

Nopp140 is the closest gene in Drosophila spp. to TCOF1 in humans. In Drosophila melanogaster, Nopp140 maps to the left arm of chromosome 3 proximal to the centromere in cytological region $78 \mathrm{~F} 4$. Two protein isoforms
Fig. 1 The second exon of Nopp140 gene contains multiple overlapping repeat patterns unique to the Drosophila melanogaster species. a Schematic representation of a region within the second exon (1650 bp in length) of Nopp140 gene that contains three repeat patterns: repeat pattern $\mathrm{P}$ with five 48 -base bp repeats, repeat pattern $\mathrm{P}^{\prime}$ with three 96 bp repeats, and region $\mathrm{P}^{\prime \prime}$ with three 96 bp repeats different from that of pattern $\mathrm{P}^{\prime}$. Nopp140 genomic regions for two isoforms of Nopp140 protein, Nopp140-True and Nopp140-RGG, are shown (solid blocks are exons and lines are introns). b Complete protein sequence of Drosophila Nopp140-True and Nopp140-RGG are shown. The beginning and ending sequence of the common second exon are highlighted in blue. Within the second exon, alternating basic (containing lysine and arginine) and acidic (containing aspartate and glutamate) motifs are highlighted in green and yellow respectively. The N-terminus (first exon which is common to both protein isoforms) and C-terminus (which is unique to each protein isoform) are highlighted in grey. The entire repeat pattern $\mathrm{P}^{\prime}$ region comprising three 96-bp repeats is marked by brackets. c Sequence alignment of Drosophila melanogaster Nopp140 gene and Nopp140-like protein coding genes from seven other Drosophila species. Shown here is the alignment of Nopp140 second exon containing the three repeat patterns $\mathrm{P}, \mathrm{P}^{\prime}$, and $\mathrm{P}^{\prime \prime}$. The first nucleotide of each repeat region is indicated by an arrow, the nucleotide number is in parenthesis (numbering begins at the first nucleotide of the second exon), and the arrowhead indicates the end of all repeats

arise by alternative splicing. Nopp140-True contains 686 amino acids, while Nopp140-RGG contains 720 residues (Waggener and DiMario 2002). Both isoforms localize to nucleoli and CBs when expressed exogenously as GFP fusions in transgenic embryos, larvae, and adults (McCain et al. 2006). The mRNA encoding Nopp140-True consists of four exons: exon 1 encodes the LisH domain as in human NOLC1 and treacle. While the large central domains in human NOLC1 and treacle are encoded by several exons, the large central domain in Drosophila Nopp140-True consists of 16 repeating basic-acidic units, all encoded by exon 2. The carboxyl terminus of Nopp140-True is encoded by exons 3 and 4 ; this domain is $65 \%$ identical to the carboxyl terminus of human NOLC1 over a 94-amino acid stretch. Therefore, we consider Nopp140-True to be the canonical orthologue of vertebrate Nopp140 (Waggener and DiMario 2002). The second isoform in Drosophila, Nopp140-RGG, is encoded by the same two exons 1 and 2, and is thus identical to Nopp140-True in its first 583 amino acid residues. Alternative splicing, however, generates a distinctly different carboxyl domain rich in glycine and arginine residues that form repeating RGG tri-peptide motifs (see Fig. 1b). Similar Nopp140-like proteins with RGG carboxyl domains have been reported in Trypanosoma brucei, Trypanosoma cruzi, and in Leishmania major (Kelly et al. 2006). RGG motifs are common to many RNA-associated proteins (Raman et al. 2001).

The large central domains of Nopp140 and treacle display low amino acid complexity, and are intrinsically disordered, a common feature for RNA chaperones (Dyson and Wright 2002; Tompa and Csermely 2004). Here we describe several 


\section{a}

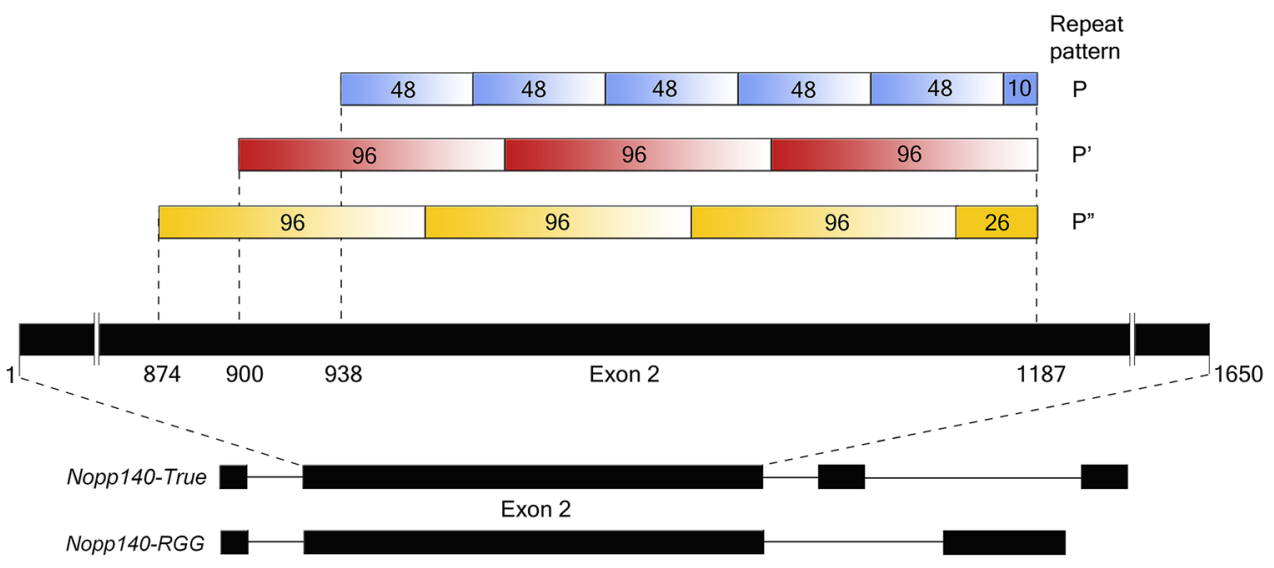

b Nopp140-True

MTDLLKIADAIVLEYLQSKDKNLAKVFQQKTKAASVAKSSPKLSEILQFYQTKSPKKIPAIKATAGDSSEDSDSDSESDAAPKKPATAPALTNGKAVKKAASSTS EDSDSEEEKKPAAKATPAKAVGKKAKSSSEDSSSEEEAPKKAAPVKAPPAKAAPAKKVESSSEDSSSEEEPAKPAVKATTTKVAPAKKADSSSEESSSDEET KPAAKPVAKAAPAKKAASSSEESDSDDEPAAKKPAVQPAAKPAPKAAASSSEDSSSEEEVKPAAKSAAKLAPAKKGASSSDDSSSEDEAPKKAATLAKPISK AAPTKKADSSTEDSSSEDDAPKKV[APAKATPAKAIPAKKAASSDDSSSEEEAPKKAAPAKATPAKAPPAKKAASSDDSSSEEEAPKKAAPAKATPAKATPA KKAASSDDSSSEEEAPKK]AAAPAKATPAKKAKSSSEDSDSDEEEAPKKPAKAVAKAASSEDSDSSEDEKPAKAAPKALAKSAKAASSDSDDSSDEETPAV KPAVKKTAAPAKKADSSSDESDSGEESGEVKPNSATNGNEKTAQKRKFSGGDQDEATPNKKYNNFVKSGEQQKNDFTSTPNNTFSRNHNMNNSGGGSGR RSPFRRVRTEDVVDSRVQDMSFEAKKNAAGSWGERANKDLKHTRGKSFKHEKTKKKRGSYRGGQIDVGVNSIKFD

Nopp140-RGG

MTDLLKIADAIVLEYLQSKDKNLAKVFQQKTKAASVAKSSPKLSEILQFYQTKSPKKIPAIKATAGDSSEDSDSDSESDAAPKKATAPALTNGKAVKKAASSTS EDSDSEEEKKPAAKATPAKAVGKKAKSSSEDSSSEEEAPKKAAPVKAPPAKAAPAKKVESSSEDSSSEEEPAKPAVKATTTKVAPAKKADSSSEESSSDEET KPAAKPVAKAAPAKKAASSSEESDSDDEPAAKKPAVQPAAKPAPKAAASSSEDSSSEEEVKPAAKSAAKLAPAKKGASSSDDSSSEDEAPKKAATLAKPISK AAPTKKADSSTEDSSSEDDAPKKV[APAKATPAKAIPAKKAASSDDSSSEEEAPKKAAPAKATPAKAPPAKKAASSDDSSSEEEAPKKAAPAKATPAKATPA KKAASSDDSSSEEEAPKK]AAAPAKATPAKKAKSSSEDSDSDEEEAPKKPAKAVAKAASSEDSDSSEDEKPAKAAPKALAKSAKAASSDSDDSSDEETPAV KPAVKKTAAPAKKADSSSDESDSGEESGEVKPNSATNGNEKTAQKRKFSGGDQDEATPNKKYNNFVKSGEQQENDFKKHNNGRGGRGGSGFSGRPDRST WETNKFNGEGGGDGGGFKKIGDRKSFGGFDNNQRGGRGGGGRGGGGGFGGRGGGGRGGGGGFGGRGGGGRGGGGFGGRGGRGGGGRGGGFGNKS FDSSAPKQNKKITFDN

C

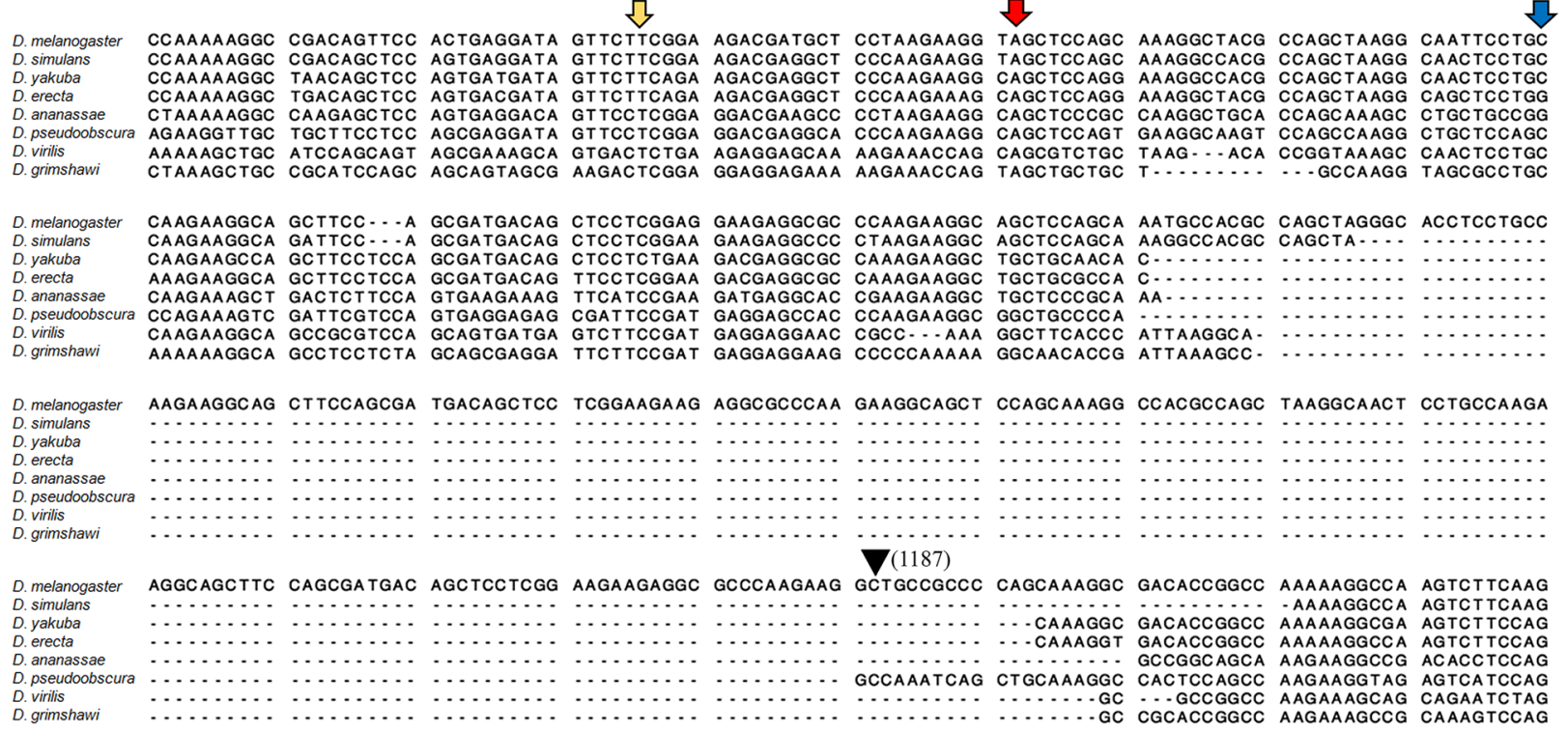


repeating but overlapping amino acid sequence patterns within the large central domain of the Nopp140 isoforms in Drosophila melanogaster. We compare differences in these patterns within other Drosophila species, and we describe two Nopp140 alleles in Drosophila melanogaster, Nopp140Long and Nopp140-Short. The two alleles differ by exactly 96 bps that encode a 31-amino acid repeat unit within the central domain. Both alleles seem to be functional as there are no apparent adverse phenotypes in flies expressing one or the other allele. The significance of this polymorphism lies in discerning the properties and functional interactions of the large central domain of metazoan Nopp140 orthologues in rDNA transcription and pre-rRNA processing.

\section{Materials and methods}

\section{Fly lines}

Flies were obtained from the Bloomington Drosophila Stock Center (Indiana University) and maintained in the laboratory at room temperature on standard cornmeal medium. The following fly stocks were used: $w^{1118}$ (Bloomington stock 3605), Daughterless-GAL4 (Da-GAL4; Bloomington stock 8641), Oregon- $R-C$ (Bloomington stock 5), Canton-S (Bloomington stock 64349), $T M 3 / E t^{50}$ (Bloomington stock 64349), TM3/Sb (Bloomington stock 4534), $\mathrm{CyO} / \mathrm{Sp}^{\mathrm{l}}$ (Bloomington stock 4199), and the Nopp140 gene deletion line, KO121 (He et al. 2015).

\section{Genomic DNA extraction}

Healthy well-fed flies were frozen at $-80{ }^{\circ} \mathrm{C}$ for $10 \mathrm{~min}$ and subsequently homogenized in $100 \mathrm{mM}$ Tris- $\mathrm{HCl}(\mathrm{pH}$ 7.5), $100 \mathrm{mM}$ EDTA, $100 \mathrm{mM} \mathrm{NaCl}$, and 0.5\% SDS. Following $30 \mathrm{~min}$ incubation at $70{ }^{\circ} \mathrm{C}$, genomic DNA was precipitated in 1:2 ratio of $5 \mathrm{M} \mathrm{KOAc:} 6 \mathrm{M} \mathrm{LiCl}$ on ice for
$10 \mathrm{~min}$. The precipitated genomic DNA was purified using phenol-chloroform extraction, followed by ethanol precipitation. The DNA pellet was suspended in deionized water, and the DNA concentration was measured by a NanoDrop 1000 Spectrophotometer.

\section{PCR amplification and band analysis}

PCR reactions were performed in $25-\mu \mathrm{L}$ total volume containing 50-100 ng (unless otherwise specified) of genomic DNA, $0.40 \mu \mathrm{M}$ of each primer (Table 1), $0.160 \mathrm{mM}$ of each dNTP, $0.30 \mathrm{mM}$ of $\mathrm{MgCl}_{2}, 0.5 \times$ Phusion GC Buffer, and 0.40 unit of Phusion high-fidelity DNA polymerase (M0530S, New England BioLabs). Amplification was performed in a BIO-RAD C1000 Thermal Cycler with the following thermal cycling conditions: 2 min initial denaturation at $95{ }^{\circ} \mathrm{C}$, followed by 34 cycles of denaturation for $30 \mathrm{~s}$ at $95^{\circ} \mathrm{C}$, annealing for $30 \mathrm{~s}$ at varying temperatures as per the primer pairs, and elongation at $72{ }^{\circ} \mathrm{C}$ for varying lengths of time depending upon amplicon sizes, followed by $5 \mathrm{~min}$ at $72{ }^{\circ} \mathrm{C}$. Phusion polymerase was used for all genomic PCRs. For the PCRs in Fig. $3 b$, the $25-\mu \mathrm{L}$ reaction volume contained 1.0 unit of Taq DNA Polymerase (MO267S, New England BioLabs), $1 \times$ ThermoPol Buffer, $0.40 \mu \mathrm{M}$ of each primer, $0.160 \mathrm{mM}$ of each $\mathrm{dNTP}$, and $0.5 \mu \mathrm{L}$ of gel extracted DNA fragments as templates. The PCR products were resolved on $1 \%$ agarose gels and imaged by ChemiDoc XRS+ system (Bio-Rad Laboratories). Image Lab ${ }^{\text {TM }}$ Software 6.0.1 (BioRad Laboratories) was used to quantify band intensities followed by Student's $\mathrm{t}$ test analysis on Microsoft Excel to obtain $p$ values.

\section{DNA sequencing and sequence analysis}

Following phenol-chloroform gel extraction and ethanol precipitation, the PCR products were sequenced with an

Table 1 Primer sequences and PCR conditions

\begin{tabular}{|c|c|c|c|}
\hline Primer pairs & $\begin{array}{l}\text { Annealing tem- } \\
\text { peratures }\left({ }^{\circ} \mathrm{C}\right)\end{array}$ & Elongation times & Sequences \\
\hline I & 61.0 & $1 \mathrm{~min}$ & $\begin{array}{l}\text { Forward: 5' ATGACAGACCTGCTAAAGATAGCC } \\
\text { Reverse: 5' AAACTCTTAGCAGGAGCCAACTTG }\end{array}$ \\
\hline II & 61.0 & $1 \mathrm{~min}$ & $\begin{array}{l}\text { Forward: 5' GTCGCAAGTTGGCTCCTGCTAAGA } \\
\text { Reverse: 5' AACATGCATAAGCGGACTGCGG }\end{array}$ \\
\hline III & 61.0 & $1 \mathrm{~min}$ & $\begin{array}{l}\text { Forward: 5' CATCTAAAATTATCCGCAGTCCGC } \\
\text { Reverse: 5' CTAGTCAAATTTTATTGAATTGACTCCTACG }\end{array}$ \\
\hline IV & 57.0 & $1 \min 30 s$ & $\begin{array}{l}\text { Forward: 5' CGGCAAGGCTGTCAAAAAGGCA } \\
\text { Reverse: 5' TTCTCATTGCCATTGGTAGC }\end{array}$ \\
\hline V & 68.0 & $25 \mathrm{~s}$ & $\begin{array}{l}\text { Forward: 5' GTCGCAAGTTGGCTCCTGCTAAGA (same as II-forward primer) } \\
\text { Reverse: 5' GGTGGAATTCGTCTTCGCTTGAAGACTTGGCCT }\end{array}$ \\
\hline Intronic & 57.0 & $1 \min 45 \mathrm{~s}$ & $\begin{array}{l}\text { Forward: 5' ATCTGCGTCCTCCTGATC } \\
\text { Reverse: 5' AACATGCATAAGCGGACTGCGG (same as II-reverse primer) }\end{array}$ \\
\hline
\end{tabular}


ABI 3130XL Genetic Analyzer (Applied Biosystems) using BigDye Terminator Cycle Sequencing kit v.3.1. The forward and reverse primers used for PCR amplification (Table 1) were used as primers to sequence the PCR products in both forward and reverse directions. Sequences were analyzed and aligned using CLC Sequence Viewer (QIAGEN Bioinformatics).

\section{Data availability}

All fly stocks obtained through the Bloomington Drosophila Resource Center can be purchased from the center. The Noppl40 gene deletion line, KO121, is available upon request. Sequences of all primers used in this study are provided in Table 1. Supplemental figures that include raw sequence reads are available in figshare.

\section{Results}

\section{Repeat sequences within the Nopp 140 second exon}

The large central domain of both Nopp140 isoforms in Drosophila melanogaster consists of several alternating basic and acidic motifs. This domain is encoded by the second exon of the Nopp140 gene (Flybase CG7421; FBgn0037137). We found a large segment within this second exon that can be displayed as three distinct but overlapping repeat patterns; we designate the patterns as $\mathrm{P}^{\prime \prime}, \mathrm{P}^{\prime}$, and P (Fig. 1a). The repeat patterns begin, respectively, at base pairs 874 , 900, and 938 with these numbering designations starting at the first base pair of the second exon (Fig. 1a). Repeat pattern $\mathrm{P}^{\prime}$ consists of three tandem repeats of $96 \mathrm{bp}$ each, and repeat pattern $\mathrm{P}^{\prime \prime}$ consists of three 96-bp tandem repeats and a fourth incomplete repeat of $26 \mathrm{bp}$. On the other hand, repeat pattern $P$ has five tandem repeats of 48 bp followed by a sixth incomplete repeat of $10 \mathrm{bp}$.

These repeat patterns contribute to the alternating basic (green) and acidic (yellow) motif pattern within the central domain of both Nopp140 isoforms in Drosophila melanogaster (Fig. 1b). For instance, the 96 bp repeat sequence in the $\mathrm{P}^{\prime}$ pattern encodes basic residues (lysine) in its first half and acidic residues (glutamate and aspartate) along with serine residues in the second half: APAKATPAKAIPAKKAASSDDSSSEEEAPKK. These serine residues are likely phosphorylated by casein kinase type II enzymes (Meier 1996) to contribute to the acidic properties of the region.

When comparing the second exon of Nopp140 from Drosophila melanogaster with Nopp140 genes from other Drosophila species (D. simulans, D. yakuba, D. erecta, $D$. ananassae, D. pseudoobscura, D. virilis, and D. grimshawi), we found that only D. melanogaster carries the repeating patterns $\mathrm{P}^{\prime \prime}, \mathrm{P}^{\prime}$ and $\mathrm{P}$. The other species carry roughly onethird of the entire repeat segment (Fig. 1c). For instance, while $D$. melanogaster and $D$. simulans are closely related species, $D$. simulans lacks 195 bps encoding 65 amino acids, essentially the latter two $\mathrm{P}^{\prime}$ repeats as compared to $D$. melanogaster. This suggests that a single $\mathrm{P}^{\prime}$ repeat is functionally sufficient for Nopp 140 proteins in the other Drosophila species, and that the extra repeats may have duplicated independently in D. melanogaster.

\section{Nopp140-Long and Nopp140-Short alleles in Drosophila melanogaster}

Here we describe a polymorphism in the Nopp140 gene of Drosophila melanogaster that involves the number of $\mathrm{P}^{\prime}$ pattern repeats described in the previous section. The allele with two repeats is referred to as Nopp140-Short, while the allele with three repeats is referred to as Nopp140-Long. Both alleles appear to be functional: fly lines homozygous for one or the other allele show no discernible abnormality. We investigated five fly lines $\left(w^{1118}\right.$, Oregon- $R$ - $C$, Canton$S$, Daughterless-GAL4, and $T M 3 / E t^{50}$ ). For each fly line, we used three PCR primer pairs I, II, and III (Table 1) to amplify the Nopp140 gene region from genomic DNA extracted from forty adult flies. Together, the three primer pairs covered the entire Nopp140 gene (Fig. 2a). While primer pairs I and III each produced a single PCR product of expected size (1046 bp and $1280 \mathrm{bp}$, respectively) in all five fly lines, primer pair II generated either one or two PCR products ( $881 \mathrm{bp}$ and/or $977 \mathrm{bp}$ ) depending on the fly line, more specifically, the long or short alleles (Fig. 2a). The $w^{1118}$ fly line produced only the short PCR product of $881 \mathrm{bp}$, indicating this stock is homozygous for Nopp140Short. Oregon-R-C, Canton-S, and Daughterless-GAL4 (DaGAL4) fly lines produced the 977-bp PCR product. These lines are therefore homozygous for Nopp140-Long. Primer pair II amplified both PCR products of $881 \mathrm{bp}$ and $977 \mathrm{bp}$ from genomic DNA isolated from the $T M 3 / E t^{50}$ fly stock. Thus, this stock is heterozygous for the Nopp140-Long and Nopp140-Short alleles (Fig. 2a). Subsequent work with a Nopp 140 gene deletion (He et al. 2015) balanced with TM3 showed that this TM3 balancer chromosome contains the Nopp140-Short allele (Supplemental Figure S1).

Sequencing the various PCR products obtained from the five fly lines (Fig. 2a) revealed that the Nopp 140-Short allele consists of two $\mathrm{P}^{\prime}$ repeats (1 and 2), whereas the Nopp140Long allele contains three $\mathrm{P}^{\prime}$ repeats $(1,2$, and 3$)$, all of which are exactly 96 bp in length. Each repeat encodes a 31-amino acid peptide sequence, with functional codons starting with the second $n t$ within the repeat thus leaving 2 nts at the end of the repeat (see Fig. 2b). 


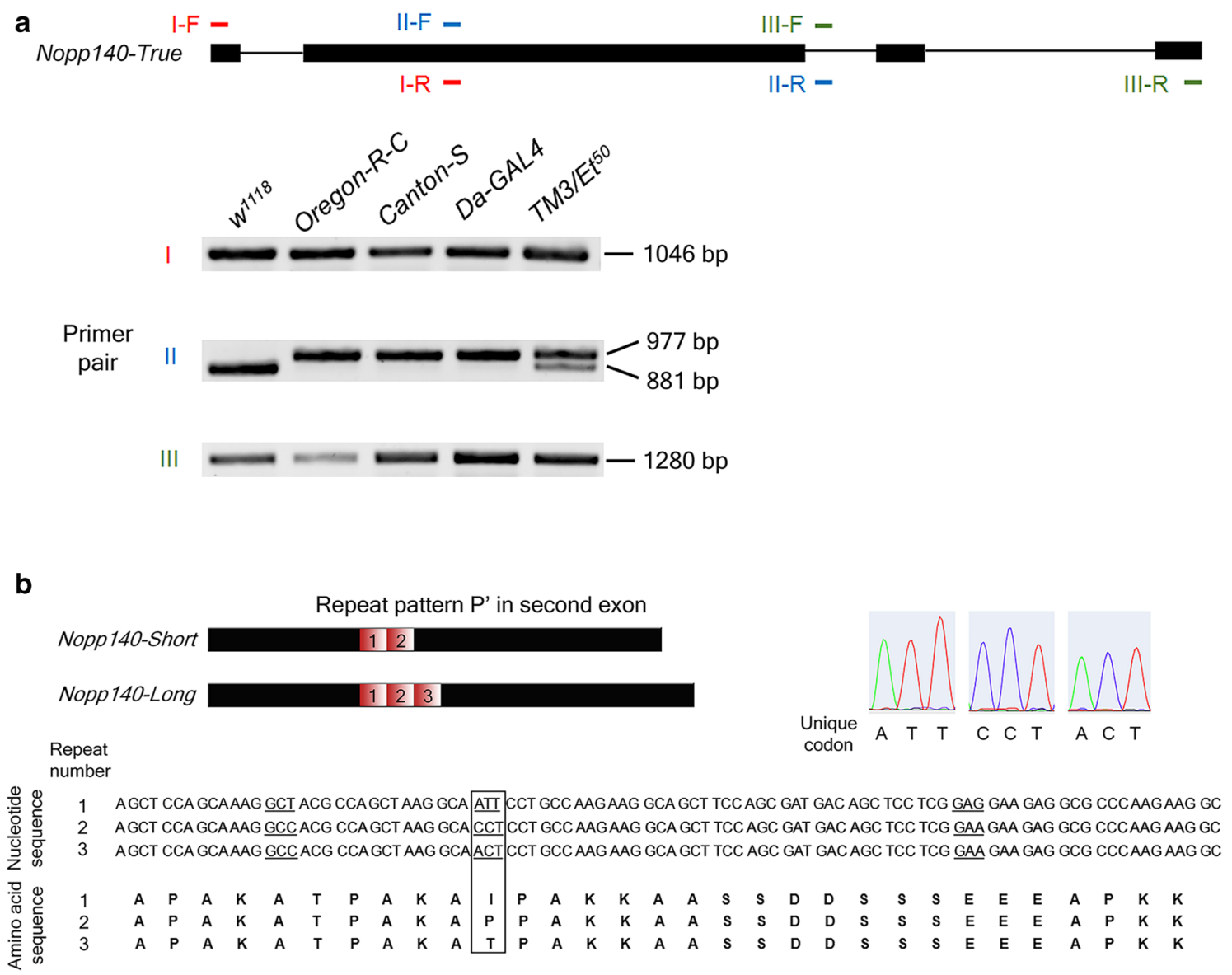

Fig. 2 Two Nopp140 alleles, Nopp140-Short and Nopp140-Long, found in Drosophila melanogaster. a PCR amplification of Nopp140 genomic DNA region (from start to stop codons for Nopp140-True isoform) in five Drosophila fly lines $(n=40 ; 20$ adult male flies and 20 adult female flies): $w^{1118}$, Oregon- $R-C$, Canton- $S$, DaughterlessGAL4 (Da-GAL4), and TM3/Et ${ }^{50}$, using three primer pairs, I, II and III ( $F$ forward, $R$ reverse). b Nopp140-Short and Nopp140-Long

The nucleotide sequence of the three repeats is identical except at three codons (codons 5, 11, and 25; underlined in Fig. 2b). Codon 5 encodes alanine in all three repeats using either GCT or GCC. Codon 25 encodes glutamate in all three repeats using either GAG or GAA. Interestingly, codon 11 encodes amino acids that are unique to each repeat; specifically, repeat 1 uses ATT encoding isoleucine, repeat 2 uses CCT encoding proline, and repeat 3 uses ACT encoding threonine (boxed enclosure in Fig. 2b; the sequencing chromatograms for these unique codons are provided). Thus, we can distinguish among the three repeats of the $\mathrm{P}^{\prime}$ pattern using these particular codons. While sequencing the PCR primer pair II products, we simultaneously sequenced the PCR products obtained with primer pairs I and III; the sequences for these PCR alleles have two and three repeats respectively, corresponding to repeat pattern $\mathrm{P}^{\prime}$, with the repeats numbered 1,2 and 3 in the $5^{\prime}-3^{\prime}$ direction on the coding strand. The nucleotide sequences of the three repeats of pattern $\mathrm{P}^{\prime}$ and the corresponding amino acid sequences for each repeat are shown. The codons at which the three repeats differ are underlined, and within the box are the codons that encode for variable amino acids that are unique to each repeat

products were identical among all five fly lines examined (Supplemental Figure S2).

\section{Preferential amplification of Nopp140-Long allele in genomic PCRs}

In our PCR analyses of fly lines heterozygous for the Nopp140-Long and Nopp140-Short alleles, such as in $T M 3 / E t^{50}$ or $T M 3 / S b$, we consistently observed the long PCR product in greater abundance compared to the short product. We used primer pairs II and IV to amplify short and long products from the $T M 3 / E t^{50}$ fly line (see Figs. 2a, $3 \mathrm{a}$, respectively), and primer pair IV to amplify short and long products from the TM3/Sb fly line (Fig. 3a). We had similar observations using genomic DNA extracted from the F1 progeny ( $D a-G A L 4 /+, n=40)$ using primer pair IV 
a

Repeat pattern $\mathrm{P}^{\prime}$

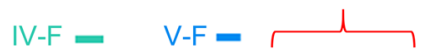

Nopp 140 second exon

$\mathrm{V}-\mathrm{R}=\quad \mathrm{IV}-\mathrm{R}=$

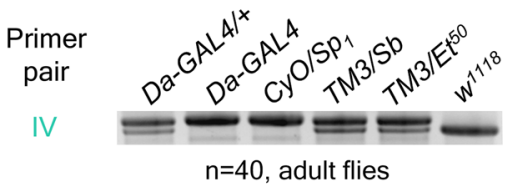

b

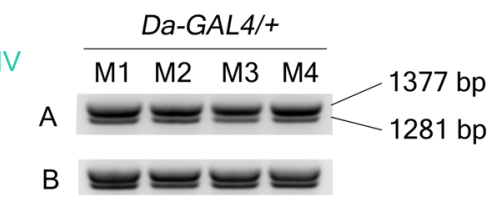

C

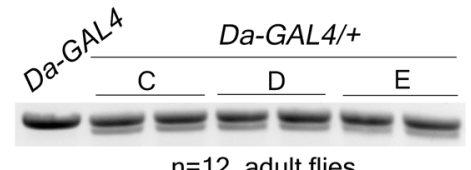

primers

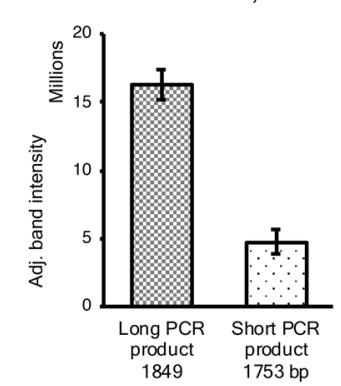

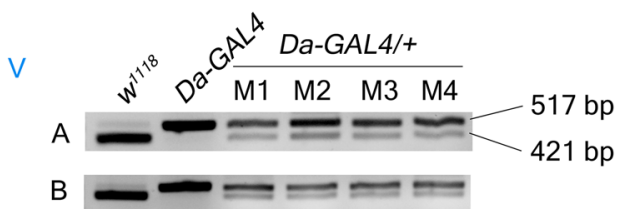

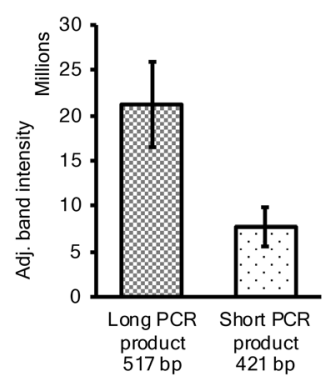

Fig. 3 Nopp140-Long allele is preferentially amplified in genomic PCRs. a PCR-amplified Nopp140 second exon containing repeat pattern $\mathrm{P}^{\prime}$ in different Drosophila melanogaster fly lines $(n=40 ; 20$ adult male flies and 20 adult female flies) using two different primer pairs, IV and V ( $F$ forward, $R$ reverse). Primer annealing sites on the Nopp140 second exon are indicated in the schematic diagram. b Primer pairs IV and $\mathrm{V}$ were used to amplify the repeat pattern $\mathrm{P}^{\prime}$ from $\mathrm{F} 1$ heterozygotes (Da-GAL4/+) obtained from reciprocal crosses, (indicated as gels A and B) between Da-GAL4 and $w^{111}$ flies. Two PCR products of $96 \mathrm{bp}$ difference were amplified indicating presence of both long and short Nopp140 alleles. M1-M4 are PCR samples in which genomic DNA extracted from single adult male flies was used as template. Quantifications of the band intensities of the long and short PCR products for the single male genomic PCRs are included. An unpaired one-tailed

$t$ test with unequal variance was performed; $p$ values were $2.1 \mathrm{E}-07$ and 4.6E-06 for PCRs with primer pairs IV and V, respectively). c PCR-amplified Nopp140 second exon containing repeat pattern $\mathrm{P}^{\prime}$ in F1 heterozygotes $D a-G A L A /+(n=12$ adult male flies $)$ obtained from three separate crosses (indicated as gel lanes C, D, and E) between $D a$ GALA and $w^{1118}$ flies, and from individual third instar larvae (indicated as gel lanes L1-L3) obtained from reciprocal cross B, using an intronic primer pair that spans the entire Nopp140 second exon. Quantification of the band intensities of the long and short PCR products from the adult male genomic PCR and the single third instar larva genomic PCR are included. An unpaired one-tailed $t$ test with unequal variance was performed; $p$ values were $1.5 \mathrm{E}-09$ and $2.4 \mathrm{E}-4$ for PCRs using genomic DNA of twelve adult flies and single third instar larvae respectively 
(Fig. 3a). These F1 progeny were obtained from a cross between the Da-GAL4 stock (homozygous for Nopp140Long) and the $w^{1118}$ stock (homozygous for Nopp140Short). The Da-GAL4 transgene resides on the third chromosome along with Nopp140-Long. Since the Da-GAL4 transgene is marked with mini-white ${ }^{+}$, we can track this particular chromosome in subsequent crosses. We obtained the same unequal product abundance between long and short products when we performed single fly PCR analyses. We used primer pairs IV and V to amplify genomic DNA extracted from single $D a-G a l 4 /+$ F1 adult male flies (Fig. 3b). The long PCR product was always in greater abundance compared to the short product.

Since we were dealing with repeat sequences, we considered the possibility that the overabundance of the long product was a PCR artifact. Therefore, we amplified the repeat region with primer pairs that were set further away from the repeats and within the two introns flanking the second exon; increasing distance of the primer annealing sites relative to the repetitive sequence is known to improve amplification of major PCR products and significantly reduce undesired PCR artifacts (Hommelsheim et al. 2014). With these intronic primers, we expected equal amplification of the long and short PCR products from heterozygous flies. However, the long PCR product was still more abundant than the short PCR product when using genomic DNA extracted from both male adult flies $(n=12)$ from three separate crosses, C, D, and E, and from a set of three single third instar larva, L1-L3 (Fig. 3c).

Based on these results, we considered the possibility of an in vivo gene conversion event occurring in the heterozygous flies whereby an insertion of a 96 bp repeat into the Nopp140-Short alleles would increase the abundance of the Nopp140-Long alleles. This could explain the overabundance of the long PCR product in the heterozygous genomic PCRs. In order to test for this possibility, we amplified the second exon of Nopp 140 from a mixture of genomic DNAs containing equal amounts (50 ng each) from $w^{1118}$ flies (homozygous for Nopp140-Short allele) and Da-GAL4 flies (homozygous for Nopp 140Long allele) using the same intronic primers. This in vitro mixing should closely mimic the genomic DNA sample from heterozygous flies, and eliminate the possibility of any gene conversion events. Once again however, the long PCR product was more abundant than the short PCR product (Fig. 4a) indicating that this is simply a PCR artifact, but one that is consistent and predictable.

In summary, we report a PCR phenomenon in which the longer genomic fragment with repeats 1,2 and 3 was preferentially amplified compared to the shorter genomic fragment with two repeats 1 and 2, despite equal amounts of the Nopp140-Short and the Nopp140-Long alleles present as templates. We never observed the opposite result a
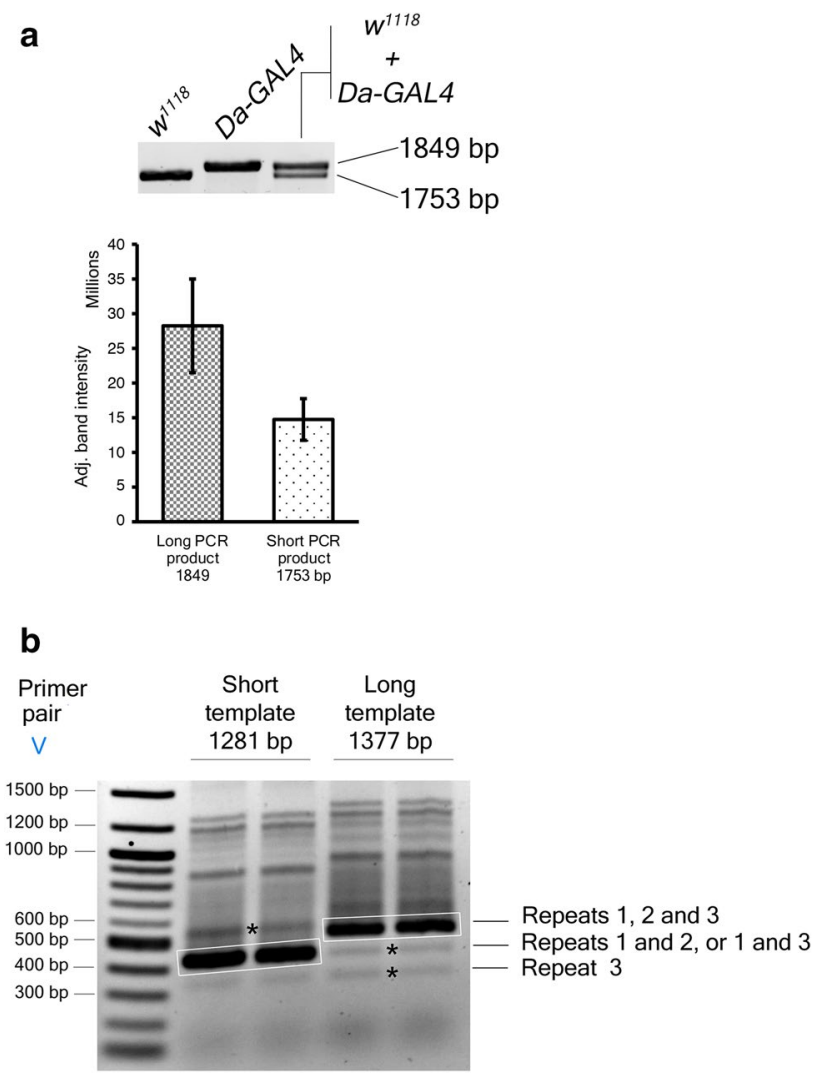

Fig. 4 PCR of repetitive DNA sequences generates artifacts. a Genomic PCRs using an intronic primer pair and one of the three templates: $50 \mathrm{ng}$ of $w^{1118}$ genomic DNA (contains only the Nopp140Short allele), $50 \mathrm{ng}$ of Da-GAL4 genomic DNA (contains only the Nopp140-Long allele), or a mixture of $50 \mathrm{ng}$ each of $w^{1118}$ and $\mathrm{Da}$ GAL4 genomic DNA (contains both Nopp140-Short and Nopp140Long alleles in equal quantities). Two PCR products of 96 bp difference, $1849 \mathrm{bp}$ and $1753 \mathrm{bp}$, were amplified among which the longer one was over amplified in PCR with the mixed genomic DNA sample $\left(D a-G A L A+w^{1118}\right)$. An unpaired one-tailed $t$ test with unequal variance was performed for the mixed genomic PCR sample $(n=5$ reactions); a $p$ value of 0.0040 was obtained. b PCR using primer pair $V$ amplified the repeat pattern $\mathrm{P}^{\prime}$ from either a 1281-bp short template DNA containing two 96-bp repeats or a 1371-bp-long template DNA containing three 96-bp repeats. The bands enclosed in white boxes are the major PCR products of expected sizes, and the undesired fragments that were also sequenced and analyzed in this study are indicated by asterisks

where the short product was more abundant than the long product.

\section{PCR amplification of undesired fragments with repetitive DNA sequences}

Studies have shown that amplification of DNA with repetitive sequences can produce an array of undesired fragments with variable number of repeats (Hommelsheim et al. 2014). Indeed, upon amplification of the repeat pattern $\mathrm{P}^{\prime}$ with primer pair V, PCR products 
of variable sizes were amplified producing a laddering effect (Fig. 4b). There were undesired PCR products both longer and shorter than the major PCR product (boxed in Fig. 4b). Surprisingly, when using a short DNA fragment of $1281 \mathrm{bp}$ (the short template) that contained two $\mathrm{P}^{\prime}$ repeats (1 and 2) as template, sequencing analysis confirmed that one of the higher molecular weight PCR fragments (marked by an asterisk in Fig. 4b) contained three $\mathrm{P}^{\prime}$ repeats: 1,2 , and 3 (in this order $5^{\prime}-3^{\prime}$, each distinguishable by the unique codons described earlier; similar to results shown in Supplemental Figure S4). Likewise, when using the long template (1377 bp) that had three $\mathrm{P}^{\prime}$ repeats $(1,2$, and 3$)$, one of the lower molecular weight PCR products contained two $\mathrm{P}^{\prime}$ repeats 1 and 2, another had two $\mathrm{P}^{\prime}$ repeats 1 and 3, and yet another had either repeat 1 or 3 (marked by asterisks in Fig. 4b; see Supplemental Figure S3). The results again suggest that the undesired PCR products with variable $\mathrm{P}^{\prime}$ pattern repeats arise as amplification artifacts.

\section{Discussion}

Vertebrate $\mathrm{NOLCl}$ and TCOF1 genes use multiple exons to encode alternating basic and acidic motifs that repeat to comprise large central domains in their protein products. The Drosophila Nopp 140 gene, however, uses a single large exon to encode a similar large central domain with alternating basic and acidic repeating motifs. We described three repeat peptide sequence patterns within this central domain. The repeats within a single pattern occur in tandem, but the three different patterns overlap with each other (Fig. 1a). One of the patterns, $\mathrm{P}^{\prime}$, consists of $96 \mathrm{bp}$ tandem repeats in Drosophila melanogaster, but other closely related Drosophila species did not carry the sequence as tandem repeats, suggesting that the repeat region in the Drosophila melanogaster Nopp140 gene may have originated relatively recently in evolutionary time.

Here we identified length polymorphisms affecting the number of $\mathrm{P}^{\prime}$ repeats. Various fly lines carry either two or three 96 bp $\mathrm{P}^{\prime}$ tandem repeats. Hence, we describe the two Nopp 140 alleles as either Nopp140-Short (two repeats) or Nopp140-Long (three repeats). The individual repeats can be identified by single nucleotide polymorphisms that exist in codon 11 (see Fig. 2b); the Short allele contains repeats 1 (ATT) and 2 (CCT), and the Long allele contains repeats 1 (ATT), 2 (CCT), and 3 (ACT). We described D. melanogaster fly lines that are homozygous for either the long allele or the short allele. Balanced fly lines can be heterozygous for both alleles.

\section{Possible PCR artifacts}

We consistently observed the second exon of Nopp140Long allele was over produced by PCR compared to that of the Nopp140-Short allele. The two alleles should be present in equal numbers in the genomic DNA extracted from heterozygous individuals, and hence PCR amplification should theoretically yield equal amounts of product from the two alleles. Initially we considered an in vivo gene conversion event in which the Nopp140-Short allele is preferentially converted to the Nopp140-Long allele as a possible explanation because we found that $w^{1118}$ genomic PCRs amplified a minor PCR product of the size expected for a long PCR product on certain occasions (Supplemental Figure S4). While we did not carry unbalanced heterozygotes $\left(D a-G A L 4 / w^{1118}\right)$ past two generations to test the stability of the long versus short alleles, we did test different larval tissues to see if amplification of the long allele was restricted to polyploid (midgut) versus diploid (brain) tissues, but we saw no difference; the long allele was always preferentially amplified regardless of the source of genomic DNA.

In a simple mixing experiment, we combined genomic DNA homozygous for Nopp140-Long with equal amounts of genomic DNA homozygous for Nopp140-Short, and observed the same preferential amplification of the long allele. This in vitro mixing experiment refutes an in vivo gene conversion event. Confusing the issue however, $w^{1118}$ genomic DNA is homozygous for Nopp 140-Short allele containing only tandem repeats 1 and 2 , but we identified minor amplicons from $w^{1118}$ genomic DNA with repeat pattern $\mathrm{P}^{\prime}$ containing tandem repeats 1, 2 and 3 (Supplemental Figure S4). Likewise, we consistently amplified (and sequenced) a 521 bp PCR product with repeat pattern $\mathrm{P}^{\prime}$ that contained tandem repeats 1, 2 and 3 (similar to the results shown in Supplemental Figure S4), although the 1281 bp template with repeat pattern $\mathrm{P}^{\prime}$ contained only tandem repeats 1 and 2 .

We have yet to explain this preferential PCR amplification of the long product versus the short product, but we may have an explanation for the amplification of short PCR products with repeats 1 and 2, 1 and 3, or a single repeat 1 (Fig. 3b) from the $1371 \mathrm{bp}$ template DNA that contained all three tandem repeats. Studies by others have shown that PCR amplification of repetitive DNA regions can generate undesired fragments of variable sizes containing haphazard combinations of repeats, similar to our observation shown in Fig. 3b. PCR products with variable repeats could potentially arise from an initial production of incomplete, singlestranded fragments that then act as mega-primers in subsequent PCR cycles. These mega-primers would then misalign with the existing templates at the repetitive sites, thereby resulting in an array of PCR fragments of sizes other than the major product (Hommelsheim et al. 2014). 


\section{Implications for long versus short Nopp140 alleles}

Earlier, we showed that partial depletion of Nopp140 in Drosophila by siRNA expression resulted in Minute-like phenotypes such as deformed wings, legs, and tergites (Cui and DiMario 2007). These phenotypes were similar to those observed with the various haplo-insufficiency Minute mutations in genes encoding ribosomal proteins, and reminiscent of the phenotypes associated with the TCS (Sæbøe-Larssen et al. 1998; Cui and DiMario 2007). Flies homozygous for either the short allele or the long allele, or flies heterozygous for the two alleles are viable and do not exhibit any of the discernible phenotypes associated with the partial depletion of the Nopp140 isoforms.

While the $\mathrm{P}^{\prime}$ repeat polymorphism does not seem to have a significant impact on the core functions of Nopp140, the protein's central domain is generally considered to be an unstructured region. Recent studies have revealed the important role of inherently disordered proteins (IDPs) in promoting phase separation to form subcellular membrane-less compartments or assemblages (Toretsky and Wright 2014), especially within nucleoli and CBs (Feric et al. 2016). Nopp140 resides within the dense fibrillar components of nucleoli and in CBs when present (Isaac et al. 1998); both nucleoli and CBs are membrane-less compartments within nuclei. The cDNA clones we have used in the past express the long versions of the two Drosophila Nopp140 isoforms; we have not worked with cDNAs encoding the short versions. Future work should determine how the large central domain of Nopp140 with variable numbers of basic-acidic repeat units may influence molecular interactions during rDNA transcription, pre-rRNA processing, snoRNP dynamics, and perhaps nucleolar and CB phaseseparations and assemblages.

Funding This study was funded by the National Science Foundation (Grant no. MCB1712975).

\section{Compliance with ethical standards}

Conflict of interest Sonu Shrestha Baral declares that she has no conflict of interest. Patrick J. DiMario declares that he has no conflict of interest.

Ethical approval All applicable international, national, and/or institutional guidelines for the care and use of animals were followed.

Open Access This article is distributed under the terms of the Creative Commons Attribution 4.0 International License (http://creativeco mmons.org/licenses/by/4.0/), which permits unrestricted use, distribution, and reproduction in any medium, provided you give appropriate credit to the original author(s) and the source, provide a link to the Creative Commons license, and indicate if changes were made.

\section{References}

Cairns C, McStay B (1995) Identification and cDNA cloning of a Xenopus nucleolar phosphoprotein, $x$ Nopp180, that is the homologue of the rat nucleolar protein Nopp140. J Cell Sci 108:3339-3347

Chen H-K, Pai C-Y, Huang J-Y, Yeh N-H (1999) Human Nopp140, which interacts with RNA polymerase I: implications for rRNA gene transcription and nucleolar structural organization. Mol Cell Biol 19:8536-8546. https://doi.org/10.1128/ MCB.19.12.8536

Cui Z, DiMario PJ (2007) RNAi knockdown of Nopp140 induces Minute-like phenotypes in Drosophila. Mol Biol Cell 18:21792191. https://doi.org/10.1091/mbc.e07-01-0074

Dyson HJ, Wright PR (2002) Coupling of folding and binding for unstructured proteins. Curr Opin Struct Biol 12:54-60. https:// doi.org/10.1016/S0959-440X(02)00289-0

Feric M, Vaidya N, Harmon TS et al (2016) Coexisting liquid phases underlie nucleolar sub-compartments. Cell 165(7):1686-1697. https://doi.org/10.1016/j.cell.2016.04.047

He F, DiMario PJ (2011). Structure and function of Nopp140 and treacle. In: Olson MOJ (ed) The nucleolus, protein reviews, 15th edn. Springer, Berlin, pp 253-278 https://doi. org/10.1007/978-1-4614-0514-6_11

He F, James A, Raje H et al (2015) Deletion of Drosophila Nopp140 induces subcellular ribosomopathies. Chromosoma 124:191208. https://doi.org/10.1007/s00412-014-0490-9

Hommelsheim CM, Frantzeskakis L, Huang M, Ülker B (2014) PCR amplification of repetitive DNA: a limitation to genome editing technologies and many other applications. Sci Rep 4:5052. https ://doi.org/10.1038/srep05052

Isaac C, Yang Y, Meier UT (1998) Nopp140 functions as a molecular link between the nucleolus and the coiled bodies. J Cell Biol 142:319-329. https://doi.org/10.1083/jcb.142.2.319

Isaac C, Marsh KL, Paznekas WA, Dixon J, Dixon MJ, Jabs EW, Meier UT (2000) Characterization of the nucleolar gene product, treacle, in Treacher Collins syndrome. Mol Biol Cell 11:3061-3071. https://doi.org/10.1091/mbc.11.9.3061

Jones NC, Lynn ML, Gaudenz K, Sakai D, Aoto K, Rey J-P et al (2008) Prevention of the neurocristopathy Treacher Collins syndrome through inhibition of p53 function. Nat Med 14:125-133. https://doi.org/10.1038/nm1725

Kelly S, Singleton W, Wickstead B, Ersfeld K, Gull K (2006) Characterization and differential nuclear localization of Nopp140 and a novel Nopp140-like protein in Trypanosomes. Eukaryot Cell 5:876-879. https://doi.org/10.1128/ec.5.5.876-879.2006

Lee C-C, Tsai Y-T, Kao C-W, Lee L-W, Lai H-J, Ma T-H et al (2014) Mutation of a Nopp140 gene dao-5 alters rDNA transcription and increases germ cell apoptosis in C. elegans. Cell Death Dis 5:e1158. https://doi.org/10.1038/cddis.2014.114

Li D, Meier UT, Dobrowolska G, Krebs EG (1997) Specific interaction between casein kinase 2 and the nucleolar protein Nopp140. J Biol Chem 272:3773-3779. https://doi.org/10.1074/ jbc.272.6.3773

Lo SJ, Lee C-C, Lai H-J (2006) The nucleolus: reviewing oldies to have new understandings. Cell Res 16:530-538. https://doi. org/10.1038/sj.cr.7310070

Mateja A, Cierpicki T, Paduch M, Derewenda ZS, Otlewski J (2006) The dimerization mechanism of Lis 1 and its implication for protein containing the LisH motif. J Mol Biol 357:621-631. https://doi.org/10.1016/j.jmb.2006.01.002

McCain J, Danzy L, Hamdi A, Dellafosse O, DiMario P (2006) Tracking nucleolar dynamics with GFP-Nopp140 during Drosophila oogenesis and embryogenesis. Cell Tissue Res 323:105115. https://doi.org/10.1007/s00441-005-0044-9 
Meier UT (1996) Comparison of the rat nucleolar protein nopp140 with its yeast homolog SRP40. Differential phosphorylation in vertebrates and yeast. J Biol Chem 271:19376-19384

Meier UT (2005) The many facets of H/ACA ribonucleoproteins. Chromosoma 114:1-14. https://doi.org/10.1007/s00412-005-0333-9

Meier UT, Blobel G (1990) A nuclear localization signal binding protein in the nucleus. J Cell Biol 111:2235-2245. https://doi. org/10.1083/jcb.111.6.2235

Meier UT, Blobel G (1992) Nopp140 shuttles on tracks between nucleolus and cytoplasm. Cell 70:127-138. https://doi. org/10.1016/0092-8674(92)90539-O

Narla A, Ebert BL (2011) Ribosomopathies: human disorders of ribosome dysfunction. Blood 115:3196-3205. https://doi.org/10.1182/ blood-2009-10-178129

Raman B, Guarnaccia C, Nadassy K, Zakhariev S, Pintar A, Zanuttin F et al (2001) $N^{\omega}$-arginine dimethylation modulates the interaction between a Gly/Arg peptide from human nucleolin and nucleic acids. Nucleic Acids Res 29:3377-3384. https://doi.org/10.1093/ nar/29.16.3377

Sæbøe-Larssen S, Lyamouri M, Merriam J, Oksvold MP, Lambertsson A (1998) Ribosomal protein insufficiency and the Minute syndrome in Drosophila: a dose-response relationship. Genetics 148:1215-1224

Sakai D, Trainor PA (2009) Treacher Collins syndrome: unmasking the role of Tcof1/treacle. Int J Biochem Cell Bio 41:1229-1232. https ://doi.org/10.1016/j.biocel.2008.10.026

Tompa P, Csermely P (2004) The role of structural disorder in the function of RNA and protein chaperone. FASEB J 18(11):1169-1175. https://doi.org/10.1096/fj.04-1584rev
Toretsky JA, Wright PE (2014) Assemblages: functional units formed by cellular phase separation. J Cell Biol 206(5):579-588. https:// doi.org/10.1083/jcb.201404124

Waggener JM, DiMario PJ (2002) Two splice variants of Nopp140 in Drosophila melanogaster. Mol Biol Cell 13:362-381. https://doi. org/10.1091/mbc.01-04-0162

Wang C, Query CC, Meier UT (2002) Immunopurified small nucleolar ribonucleoprotein particles pseudouridylate rRNA independently of their association with phosphorylated Nopp 140 . Mol Cell Biol 22:8457-8466. https://doi.org/10.1128/ MCB.22.24.8457-8466.2002

Wise CA, Chiang LC, Paznekas WA, Sharma M, Musy MM, Ashley JA et al (1997) TCOF1 gene encodes a putative nucleolar phosphoprotein that exhibits mutations in Treacher Collins Syndrome throughout its coding region. Proc Natl Acad Sci USA 94:3110 3115. https://doi.org/10.1073/pnas.94.7.3110

Yang Y, Isaac C, Wang C, Dragon F, Pogacic V, Meier UT (2000) Conserved composition of mammalian box H/ACA and box C/D small nucleolar ribonucleoprotein particles and their interaction with the common factor Nopp140. Mol Biol Cell 11:567-577. https://doi.org/10.1091/mbc.11.2.567

Publisher's Note Springer Nature remains neutral with regard to jurisdictional claims in published maps and institutional affiliations. 